\title{
Compensation of Systematic Measurement Errors in a PMU-based Monitoring System for Electric Distribution Grids
}

\author{
Paolo Attilio Pegoraro*, Kyle Brady ${ }^{\dagger}$, Paolo Castello*, Carlo Muscas*, Alexandra von Meier ${ }^{\dagger}$ \\ *Department of Electrical and Electronic Engineering, University of Cagliari, Cagliari, 09123 Italy \\ ${ }^{\dagger}$ Department of Electrical Engineering and Computer Science, University of California, Berkeley, Berkeley, CA, \\ USA
}

\begin{abstract}
The availability of accurate measurements is the prerequisite for the actual implementation of many monitoring and management applications in smart distribution networks. Phasor Measurement Units (PMUs) can provide synchronized voltage and current measurements, referred to a common time reference (usually the Coordinated Universal Time, UTC). This feature, as well as the high accuracy and reporting rate of PMUs, can be exploited for an accurate network monitoring.

At the same time, the smartness of the grid can include the possibility for the measurement system to self-detect its weak points and improve its performance. In this perspective, a technique for the estimation and the compensation of systematic errors existing in the components of a PMU-based distributed measurement system suitable for monitoring three-phase distribution networks is presented. The uncertainty induced by the components of the measurement system, mainly instrument transformers and PMUs, is included in the model, along with the uncertainty affecting the values of the network line parameters. The method exploits the possible constraints introduced by the grid topology (presence of multiple lines, injected currents, etc.) to improve the accuracy of the estimation, so that a proper compensation of the estimated errors can be allowed. The validity of the approach is verified though simulations performed on a small portion of a test medium voltage distribution grid.
\end{abstract}

Index Terms-phasor measurement units, power distribution lines, instrument transformers, voltage measurement, current measurement, measurement errors, impedance measurement.

\section{INTRODUCTION}

Sizeable changes are occurring in power distribution networks (DNs) with the advent of distributed energy sources and storage systems, particularly distributed renewables, as well as with the introduction of electric vehicles and the proposals for responsive load management and new energy markets.

This evolution asks for new approaches in the management and control of the network, requiring a more intelligent cyberphysical infrastructure for DNs that allows transforming them into "smart" DNs (SDNs). A prerequisite for advanced network operation is an upgraded and sophisticated monitoring system that can foster the transition towards network automation.

Phasor Measurement Units (PMUs), which provide synchronized measurements of voltage and current phasors, as well as frequency and rate of change of frequency (ROCOF), can represent an interesting improvement to the monitoring infrastructure. Thanks to the synchronization with respect to a common coordinated universal time (UTC) reference, their high accuracy and reporting rates, these devices are starting to play an important role in modern electric power grids, forming the basis of advanced and more effective monitoring applications. PMUs are currently employed mainly in the transmission grid, where synchrophasor measurements from PMUs are used for several applications [1]. PMU measurements can be used, for example, for state estimation [2], [3], voltage stability assessment, line parameter estimations [4]-[6] and also for off-line applications such as post-mortem analysis.

Because of their high potential, PMUs have begun to be considered for use in the monitoring and management of SDNs as well. The development of PMUs specifically conceived for this type of network encourages the use of PMUs in many distribution applications [7], such as state estimation techniques applied to DNs [8], [9]. There are research projects aiming to design low-cost PMUs [10], [11] and at a large-scale installation in DNs [12].

One interesting application that can be made feasible by the widespread use of PMUs is estimating and compensating for the systematic errors existing in the measurement system, introduced by both the Instrument Transformers (ITs) and the PMUs. Referring to transmission systems, paper [13] proposes a method that can simultaneously estimate line parameters as well as measurements. It requires the availability of accurate measurements that can be achieved by a precalibrated PMU receiving voltage and current signals from precalibrated ITs. A PMU-based approach for on-line calibration of voltage transformers has been presented in [14]. It requires the placement of multiple good quality voltage measurements (i.e. achievable again by pre-calibrated devices) at optimal locations inside the network. Paper [15] presents a framework for online bias error detection and calibration of PMU measurements in a single transmission line using density-based spatial clustering of applications with noise.

Focusing on distribution grids, limited attention has been paid to PMU application in this field. In [16], the feasibility of directly calculating impedances (of a transformer and a single line) in DNs is investigated, but device and transducer uncertainties are not considered in the model.

In this paper the main concepts of an approach aimed at estimating the DN's line parameters on the basis of PMU measurements, proposed in [17] for single-phase systems 
and extended in [18] for three-phase systems, are adapted and integrated to define an original technique to estimate the systematic errors existing in the distributed measurement system, including both ITs and PMUs. The proposed method does not require pre-calibrated measurement devices, which would become impractical in DNs. It is designed to work continuously online for three-phase unbalanced networks. It suitably exploits the different constraints that characterize the grid. Injected currents and zero injection constraints are introduced and used together with voltage drops to improve the accuracy of the estimation, so that a proper compensation of the estimated errors can be allowed. The method, which is based on a recursive linearized weighted least squares (WLS), is designed to operate on multiple branches simultaneously to improve the estimation performance. Simulations performed on a sample DN derived from the IEEE 13 bus test network are used to study the perfomance of the proposed methodology and the impact of the different constraints.

\section{Proposed METhod}

\section{A. Network and Measurement Model}

When a three-phase unbalanced distribution network is considered, a model for the generic branch $(i, j)$ can be defined as in Fig. 1. Shunt impedances are neglected as is usual in DNs due to their small values ${ }^{1}$. The model can be represented by means of the following symmetric $3 \times 3$ impedance matrix:

$$
\mathbf{Z}_{i j}=\left[\begin{array}{lll}
z_{i j, a a} & z_{i j, a b} & z_{i j, a c} \\
z_{i j, a b} & z_{i j, b b} & z_{i j, b c} \\
z_{i j, a c} & z_{i j, b c} & z_{i j, c c}
\end{array}\right]
$$

where $z_{i j, p q}=R_{i j, p q}+j X_{i j, p q}(p$ and $q \in\{a, b, c\})$. When one or two phases are not present in a branch (two-phase or single-phase branch, respectively), the branch impedance matrix is reduced accordingly and becomes $2 \times 2$ or $1 \times 1$.

The measurement system considered in the following is represented by the synchronized measurements indicated in Fig. 1: the wye-connected voltage synchrophasors $v_{i, a}, v_{i, b}$, $v_{i, c}, v_{j, a}, v_{j, b}, v_{j, c}$ at both ends of the line and the line current synchrophasors $i_{i j, a}, i_{i j, b}, i_{i j, c}$. These measurements are given by PMU devices that are assumed to be installed at each node of the monitored network or subnetwork. Each PMU can have multiple input channels and is connected to the corresponding outputs of the instrument transformers (e.g. VTs and CTs). PMU measurements can be time-aligned, thanks to the timetag associated with each measurement frame. In the following and in the simulations of Section III the measurements are considered, for the sake of simplicity, as independent of one another. This assumption is realistic when measurements from different instruments are considered. When multiple channels of the same PMU are used, independence is not guaranteed. Indeed, measured synchrophasors may present different levels of correlation depending on PMU architecture and channel coupling. For instance, a more detailed discussion about the

\footnotetext{
${ }^{1}$ If large shunt impedances were present, the models introduced in the following could be modified to include them. In this case, it would be useful to include in the measurement system also the end node current measurements for each branch.
}

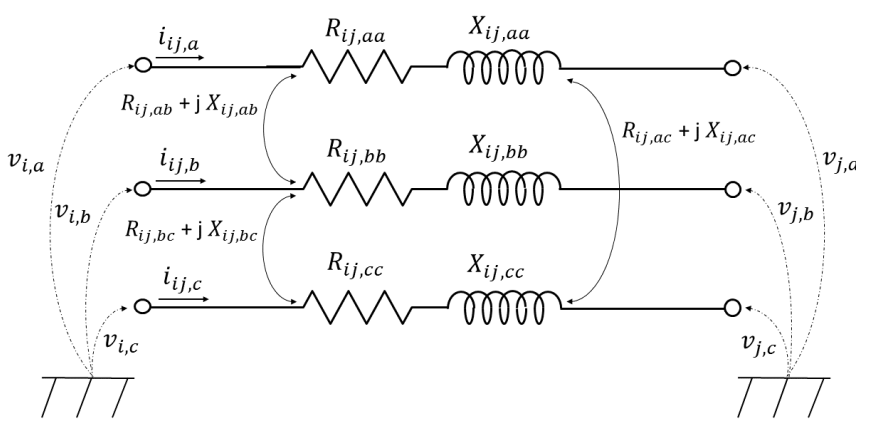

Fig. 1. A generic network branch three-phase model and its parameters.

effect of a common timebase on correlation can be found in [19]. If this kind of information is available, it can be included rather easily in the model.

Following the approach [18], the above three-phase unbalanced model is used to define a measurement model that considers all phasor measurements at a single timestamp ${ }^{2}$ and includes both systematic and random contributions to the measurement uncertainty of the modeled PMU data.

As in [18], we first consider the single-branch model and then we extend it to a set of branches, looking at the measurements synchronized across a network. Referring to Fig. 1, the voltage phasor measurements can be expressed as:

$$
v_{i, p}=V_{i, p} e^{j \varphi_{i, p}}=V_{i, p}^{r}+j V_{i, p}^{x}
$$

where $V_{i, p}$ and $\varphi_{i, p}$ (with $p \in\{a, b, c\}$ ) are, respectively, the magnitude and phase-angle measurements for phase $p$ of the $i$ th node. $V_{i, p}^{r}$ and $V_{i, p}^{x}$ are the corresponding real and imaginary parts of the phasor measurement $v_{i, p}$. Analogous symbols and definitions can be adopted for every node and, in particular, for node $j$ in the Fig. 1.

Similarly, the three current phasors of the line connecting nodes $i$ and $j$ are:

$$
i_{i j, p}=I_{i j, p} e^{j \theta_{i j, p}}=I_{i j, p}^{r}+j I_{i j, p}^{x}
$$

where the $I_{i j, p}, \theta_{i j, p}, I_{i j, p}^{r}$ and $I_{i j, p}^{x}$ have the same meaning as before (measurements of magnitude, phase angle and the corresponding real and imaginary parts of the measured synchrophasor) with the phase $p \in\{a, b, c\}$.

The measurement model requires the expression of the measurements in (2) and (3) in terms of the measurement errors as follows ( $h \in\{i, j\}$ and $p$ is always the generic phase):

$$
\begin{aligned}
v_{h, p} & =V_{h, p} e^{j \varphi_{h, p}} \\
& =\left(1+\xi_{h, p}^{s y s}+\xi_{h, p}^{r n d}\right) V_{h, p}^{R} e^{j\left(\varphi_{h, p}^{R}+\alpha_{h, p}^{s y s}+\alpha_{h, p}^{r n d}\right)} \\
i_{i j, p} & =I_{i j, p} e^{j \theta_{i j, p}} \\
& =\left(1+\eta_{i j, p}^{s y s}+\eta_{i j, p}^{r n d}\right) I_{i j, p}^{R} e^{j\left(\theta_{i j, p}^{R}+\psi_{i j, p}^{s y s}+\psi_{i j, p}^{r n d}\right)}
\end{aligned}
$$

where (as in [18]) both the systematic and random errors are explicitly indicated by the superscrits ${ }^{s y s}$ and ${ }^{r n d}$, respectively. In particular, in the first equation, $\xi_{h, p}$ and $\alpha_{h, p}$ are the errors

\footnotetext{
${ }^{2}$ the timestamp is not indicated in the following, unless strictly necessary for the clarity of presentation
} 
associated with the voltage magnitude measurement $V_{h, p}$ and the phase-angle measurement $\varphi_{h, p}$ given by a PMU. $V_{h, p}^{R}$ and $\varphi_{h, p}^{R}$ are the reference values (superscript ${ }^{R}$ from here on) of the measured quantities, which are altered by ratio and phasedisplacement errors.

In (4b), with a similar notation, the parameters $\eta_{i j, p}^{s y s}$ and $\psi_{i j, p}^{s y s}$ are the systematic ratio and phase-angle errors associated with the synchronized measurements of the three currents. As before, the superscript ${ }^{r n d}$ denotes the random counterparts of the errors. The reference quantities of the current measurements are denoted by the superscript $R$.

In (4) it is assumed that the contributions of the small relative errors, whatever their nature, in each stage of the measurement chain can be summed up since the second order products become negligible (with the uncertainties assumed in Section III-A, they are typically three orders of magnitude smaller). With this assumption, second order errors that arise whenever there is a cascade of two relative errors are not included in the model.

Separating the two error types highlights the different role that the two contributions play: the systematic error is constant across repeated measurements, while the random error changes for every observation. For this reason, the first one is considered in the following as an unknown parameter to estimate, while the second one gives the unavoidable uncertainty of the available measurements. The measurement model in (4) allows defining the parameters to be estimated by an algorithm that aims at finding the compensation parameters for voltage and current measurement chains.

\section{B. Line Voltage Drop Equations}

The estimation algorithm must rely on the synchronized measurements and on the constraints given by Kirchoff's laws. In particular, in the single-branch case, the three-phase voltage drop across the line sets the following relationships:

$$
\Delta \mathbf{v}_{i j}^{R}=\mathbf{v}_{i}^{R}-\mathbf{v}_{j}^{R}=\mathbf{Z}_{i j} \mathbf{i}_{i j}^{R}
$$

where $\mathbf{v}_{i}^{R}=\left[v_{i, a}^{R}, v_{i, b}^{R}, v_{i, c}^{R}\right]^{\mathrm{T}}$ and $\mathbf{v}_{j}^{R}=\left[v_{j, a}^{R}, v_{j, b}^{R}, v_{j, c}^{R}\right]^{\mathrm{T}}$ are the vectors of reference voltage phasors and $\mathbf{i}_{i j}^{R}=$ $\left[i_{i j, a}^{R}, i_{i j, b}^{R}, i_{i j, c}^{R}\right]^{\mathrm{T}}$ is the vector of reference current phasors ( $\mathrm{T}$ indicates the transpose operator).

Equation (5) includes the actual branch-impedance matrix that is known with a degree of uncertainty, since it is based on prior knowledge from network database or previous measurement campaigns. It is possible to rewrite (5) expressing $\mathbf{v}_{i}^{R}, \mathbf{v}_{j}^{R}$ and $\mathbf{i}_{i j}^{R}$ in terms of the measured values and the measurement errors. This amounts to making explicit the reference values from (4). They can then be written as follows:

$$
\begin{aligned}
v_{h, p}^{R} & =V_{h, p}^{R} e^{j \varphi_{h, p}^{R}}=\frac{V_{h, p} e^{j \varphi_{h, p}}}{\left(1+\xi_{h, p}^{s y s}+\xi_{h, p}^{r n d}\right)} e^{-j\left(\alpha_{h, p}^{s y s}+\alpha_{h, p}^{r n d}\right)} \\
& \simeq V_{h, p} e^{j \varphi_{h, p}}\left(1-\xi_{h, p}^{s y s}-\xi_{h, p}^{r n d}\right)\left[1-j\left(\alpha_{h, p}^{s y s}+\alpha_{h, p}^{r n d}\right)\right]
\end{aligned}
$$$$
i_{i j, p}^{R}=I_{i j, p}^{R} e^{j \theta_{i j, p}^{R}}=\frac{I_{i j, p} e^{j \theta_{i j, p}}}{\left(1+\eta_{i j, p}^{s y s}+\eta_{i j, p}^{r n d}\right)} e^{-j\left(\psi_{i j, p}^{s y s}+\psi_{i j, p}^{r n d}\right)}
$$

$$
\simeq I_{i j, p} e^{j \theta_{i j, p}}\left(1-\eta_{i j, p}^{s y s}-\eta_{i j, p}^{r n d}\right)\left[1-j\left(\psi_{i j, p}^{s y s}+\psi_{i j, p}^{r n d}\right)\right]
$$

where $h \in\{i, j\}$ as above and the last approximation in each equation is obtained by assuming both the magnitude relative errors $\xi_{i, p}^{s y s}, \xi_{i, p}^{r n d}, \xi_{j, p}^{s y s}, \xi_{j, p}^{r n d}, \eta_{i j, p}^{s y s}, \eta_{i j, p}^{r n d}$ and the phaseangle deviations $\alpha_{i, p}^{s y s}, \alpha_{i, p}^{r n d}, \alpha_{j, p}^{s y s}, \alpha_{j, p}^{r n d}, \psi_{i j, p}^{s y s}, \psi_{i j, p}^{r n d}$ much smaller than one ${ }^{3}$, as it is, since transducer and PMU errors are typically less than $1 \%$ for amplitudes and less than $1 \mathrm{crad}$ $\left(10^{-2} \mathrm{rad}\right)$.

Focusing on phase $a$ (similar expressions hold for the other phases) it is possible to rewrite the voltage drop constraint as follows, by substituting (1), (2), (3), and (6) in (5):

$$
\begin{gathered}
\left(V_{i, a}^{r}+j V_{i, a}^{x}\right)\left(1-\xi_{i, a}^{s y s}-\xi_{i, a}^{r n d}\right)\left[1-j\left(\alpha_{i, a}^{s y s}+\alpha_{i, a}^{r n d}\right)\right]+ \\
-\left(V_{j, a}^{r}+j V_{j, a}^{x}\right)\left(1-\xi_{j, a}^{s y s}-\xi_{j, a}^{r n d}\right)\left[1-j\left(\alpha_{j, a}^{s y s}+\alpha_{j, a}^{r n d}\right)\right] \\
\simeq \sum_{p \in\{a, b, c\}}\left\{\left(R_{i j, a p}+j X_{i j, a p}\right)\left(I_{i j, p}^{r}+j I_{i j, p}^{x}\right)\right. \\
\left.\quad \times\left(1-\eta_{i j, p}^{s y s}-\eta_{i j, p}^{r n d}\right)\left[1-j\left(\psi_{i j, p}^{s y s}+\psi_{i j, p}^{r n d}\right)\right]\right\}
\end{gathered}
$$

The constraint in (5), which involves only reference values and branch impedances, is instead represented in (7) as a relationship between the measured quantities and the measurement errors of both magnitude and phase angle, using the approximated expressions in (6).

Equation (7) is the complex-valued constraint of the first phase and it can be rewritten in its real and imaginary parts, after a few simplifications, as:

$$
\begin{aligned}
V_{i, a}^{r}- & V_{j, a}^{r}-\sum_{p \in\{a, b, c\}}\left(R_{i j, a p} I_{i j, p}^{r}-X_{i j, a p} I_{i j, p}^{x}\right) \simeq \\
& V_{i, a}^{r}\left(\xi_{i, a}^{s y s}+\xi_{i, a}^{r n d}\right)-V_{j, a}^{r}\left(\xi_{j, a}^{s y s}+\xi_{j, a}^{r n d}\right) \\
& -V_{i, a}^{x}\left(\alpha_{i, a}^{s y s}+\alpha_{i, a}^{r n d}\right)+V_{j, a}^{x}\left(\alpha_{j, a}^{s y s}+\alpha_{j, a}^{r n d}\right) \\
& +\sum_{p \in\{a, b, c\}}\left(-R_{i j, a p} I_{i j, a p}^{r}+X_{i j, a p} I_{i j, a p}^{x}\right)\left(\eta_{i j, p}^{s y s}+\eta_{i j, p}^{r n d}\right) \\
& +\sum_{p \in\{a, b, c\}}\left(R_{i j, a p} I_{i j, a p}^{x}+X_{i j, a p} I_{i j, a p}^{r}\right)\left(\psi_{i j, p}^{s y s}+\psi_{i j, p}^{r n d}\right) \\
& V_{i, a}^{x}-V_{j, a}^{x}-\sum_{p \in\{a, b, c\}}\left(X_{i j, a p} I_{i j, p}^{r}+R_{i j, a p} I_{i j, p}^{x}\right) \simeq \\
& V_{i, a}^{x}\left(\xi_{i, a}^{s y s}+\xi_{i, a}^{r n d}\right)-V_{j, a}^{x}\left(\xi_{j, a}^{s y s}+\xi_{j, a}^{r n d}\right) \\
+ & V_{i, a}^{r}\left(\alpha_{i, a}^{s y s}+\alpha_{i, a}^{r n d}\right)-V_{j, a}^{r}\left(\alpha_{j, a}^{s y s}+\alpha_{j, a}^{r n d}\right) \\
+ & \sum_{p \in\{a, b, c\}}\left(-X_{i j, a p} I_{i j, a p}^{r}-R_{i j, a p} I_{i j, a p}^{x}\right)\left(\eta_{i j, p}^{s y s}+\eta_{i j, p}^{r n d}\right) \\
+ & \sum_{p \in\{a, b, c\}}\left(X_{i j, a p} I_{i j, a p}^{x}-R_{i j, a p} I_{i j, a p}^{r}\right)\left(\psi_{i j, p}^{s y s}+\psi_{i j, p}^{r n d}\right)
\end{aligned}
$$

where approximations have been introduced considering the second order terms $\left(\xi_{i, a}^{s y s}+\xi_{i, a}^{r n d}\right)\left(\alpha_{i, a}^{s y s}+\alpha_{i, a}^{r n d}\right),\left(\xi_{j, a}^{s y s}+\right.$ $\left.\xi_{j, a}^{r n d}\right)\left(\alpha_{j, a}^{s y s}+\alpha_{j, a}^{r n d}\right)$, and $\left(\eta_{i j, p}^{s y s}+\eta_{i j, p}^{r n d}\right)\left(\psi_{i j, p}^{s y s}+\psi_{i j, p}^{r n d}\right)$ negligible with respect to the others.

\footnotetext{
${ }^{3}$ the approximations $(1+x)^{-1} \simeq(1-x)$ and $e^{x} \simeq(1+x)$ have been used.
} 
Equations (8), along with their phase $b$ and phase $c$ counterparts, show that these physical constraints define new equivalent measurements that depend on the unknown systematic errors and on the random measurement errors introduced by the transducer-plus-PMU system for each quantity of interest. These equations could be directly used to estimate the systematic errors, but, as mentioned above, the line parameters are not accurately known. In order to estimate such errors, we exploit the same approach as in [18], but with a different target.

The generic element of $\mathbf{Z}_{i j}$ can be expressed as:

$$
\begin{aligned}
z_{i j, p q} & =R_{i j, p q}+j X_{i j, p q} \\
& =R_{i j, p q}^{0}\left(1+\gamma_{i j, p q}\right)+j X_{i j, p q}^{0}\left(1+\beta_{i j, p q}\right)
\end{aligned}
$$

where $\gamma_{i j, p q}$ and $\beta_{i j, p q}$ ( $p$ and $q \in\{a, b, c\}$ ) are the relative deviations of the actual values of resistance and reactance parameters, respectively, from the available values $R_{i j, p q}^{0}$ and $X_{i j, p q}^{0}$ (e.g. nominal parameter values from DSO database). These deviations are unknown and take into account the lack of knowledge about the impedances while aiming at the estimation of systematic measurement errors in voltage and current. If neglected, they could have an impact on the systematic error's estimation.

Using (9) into (8), along with the expressions for the other phases, and neglecting also the second order terms involving $\gamma_{i j, p q}$ and $\beta_{i j, p q}$, it is possible to write the six equations associated with the voltage drop as the following linear problem:

$$
\begin{aligned}
& \mathbf{b}_{i j}=\left[\begin{array}{ll}
\mathbf{H}_{i j}^{\xi, \alpha} \mathbf{H}_{i j}^{\eta, \psi} \mathbf{H}_{i j}^{\gamma, \beta}
\end{array}\right]\left[\begin{array}{c}
\xi_{i j}^{s y s} \\
\boldsymbol{\alpha}_{i j}^{s y s} \\
\boldsymbol{\eta}_{i j}^{s y s} \\
\boldsymbol{\psi}_{i j}^{s y s} \\
\boldsymbol{\gamma}_{i j}^{s y j} \\
\boldsymbol{\beta}_{i j}
\end{array}\right]+\left[\begin{array}{l}
\mathbf{H}_{i j}^{\xi, \alpha} \mathbf{H}_{i j}^{\eta, \psi}
\end{array}\right]\left[\begin{array}{c}
\boldsymbol{\xi}_{i j}^{r n d} \\
\boldsymbol{\alpha}_{i j}^{r n d} \\
\boldsymbol{\eta}_{i j}^{r n d} \\
\boldsymbol{\psi}_{i j}^{r n d}
\end{array}\right] \\
& =\mathbf{H}_{i j} \mathbf{x}_{i j}+\mathbf{\epsilon}_{i j}
\end{aligned}
$$

where $\mathbf{b}_{i j}$ is the vector of equivalent measurements associated with branch $(i, j)$ and is written as (only phase- $a$ rows are explicitly reported for the sake of brevity):

$\mathbf{b}_{i j}=\left[\begin{array}{cc}V_{i, a}^{r}-V_{j, a}^{r}-\sum_{p \in\{a, b, c\}} & \left(R_{i j, a p}^{0} I_{i j, p}^{r}-X_{i j, a p}^{0} I_{i j, p}^{x}\right) \\ V_{i, a}^{x}-V_{j, a}^{x}-\sum_{p \in\{a, b, c\}} & \left(R_{i j, a p}^{0} I_{i j, p}^{x}+X_{i j, a p}^{0} I_{i j, p}^{r}\right) \\ \vdots\end{array}\right]$

$\mathbf{H}_{i j}^{\xi, \alpha}$ is the submatrix expressing the linear measurement function of these equivalent measurements with respect to voltage amplitude and phase-angle errors, while $\mathbf{H}_{i j}^{\eta, \psi}$ is the analogous matrix relating to current amplitude and phaseangle errors. The systematic deviations are collected into the subvectors:

$$
\begin{aligned}
\xi_{i j}^{s y s} & =\left[\xi_{i, a}^{s y s}, \xi_{j, a}^{s y s}, \xi_{i, b}^{s y s}, \xi_{j, b}^{s y s}, \xi_{i, c}^{s y s}, \xi_{j, c}^{s y s}\right]^{\mathrm{T}} \\
\boldsymbol{\alpha}_{i j}^{s y s} & =\left[\alpha_{i, a}^{s y s}, \alpha_{j, a}^{s y s}, \alpha_{i, b}^{s y s}, \alpha_{j, b}^{s y s}, \alpha_{i, c}^{s y s}, \alpha_{j, c}^{s y s}\right]^{\mathrm{T}} \\
\boldsymbol{\eta}_{i j}^{s y s} & =\left[\eta_{i j, a}^{s y s}, \eta_{i j, b}^{s y s}, \eta_{i j, c}^{s y s}\right]^{\mathrm{T}}
\end{aligned}
$$

$$
\boldsymbol{\psi}_{i j}^{s y s}=\left[\psi_{i j, a}^{s y s}, \psi_{i j, b}^{s y s}, \psi_{i j, c}^{s y s}\right]^{\mathrm{T}}
$$

Finally, $\quad \mathbf{H}_{i j}^{\gamma, \beta}$ is the submatrix that takes into account the line parameter deviations $\gamma_{i j}=\left[\gamma_{i j, a a}, \gamma_{i j, b b}, \gamma_{i j, c c}, \gamma_{i j, a b}, \gamma_{i j, a c}, \gamma_{i j, b c}\right]^{\mathrm{T}} \quad$ and $\boldsymbol{\beta}_{i j}=\left[\beta_{i j, a a}, \beta_{i j, b b}, \beta_{i j, c c}, \beta_{i j, a b}, \beta_{i j, a c}, \beta_{i j, b c}\right]^{\mathrm{T}}$. Details on the entries of $\mathbf{H}_{i j}^{\xi, \alpha}, \mathbf{H}_{i j}^{\eta, \psi}$ and $\mathbf{H}_{i j}^{\gamma, \beta}$ are given in Appendix A. All the unknowns associated with branch $(i, j)$ equations are included in the state $\mathbf{x}_{i j}$ along with the impedance parameter deviations.

As for the random errors, vector $\boldsymbol{\epsilon}_{i j}$ is the vector of the six equivalent random errors associated with the six equivalent measurements in (10). $\boldsymbol{\epsilon}_{i j}$ is obtained from the voltage and current random errors by a matrix multiplication, using the same submatrices $\mathbf{H}_{i j}^{\xi, \alpha}$ and $\mathbf{H}_{i j}^{\eta, \psi}$ as before.

To estimate the unknowns $\left[\xi_{i j}^{\text {sys } \mathrm{T}} \boldsymbol{\alpha}_{i j}^{\text {sys }} \boldsymbol{\eta}_{i j}^{s y s \mathrm{~T}} \boldsymbol{\psi}_{i j}^{\text {sys } \mathrm{T}}\right]^{\mathrm{T}}$, it is necessary to estimate the state $\mathbf{x}_{i j}$. The main idea is to use multiple measurement sets corresponding to different timestamps (giving us different equations (10), one for each time-tag $t$ ). In the next sections additional constraints are discussed and the estimation algorithm is presented in detail.

\section{Multiple Lines and Injection Constrains}

When considering a full set of nodes and the corresponding branches, the voltage drop along all lines must be considered, which provides additional constraints along with additional unknown parameters corresponding to all included voltage and current phasor measurements. All the measurements corresponding to time $t$ can be considered simultaneously thanks to PMU absolute time alignment.

For this reason, a new state $\mathbf{x}$ is defined as the concatenation of vectors $\mathbf{x}_{i j}$ for $(i, j) \in \Gamma$, where $\Gamma$ is the set of considered branches. For each $t$, a vector $\mathbf{b}$ and a matrix $\mathbf{H}$ are obtained by vertically concatenating $\mathbf{b}_{i j}$ and $\mathbf{H}_{i j}$ for $(i, j) \in \Gamma$, thus defining the overall estimation problem.

In addition, Kirchoff's Current Law applied at each node can help define new constraints. In this paper, we assume that current injection measurements are available. In the following, current synchrophasors are condsidered injected at a given node $j$ as in Fig. 2.

The additional three-phase constraint given by the measured injection $\mathbf{i}_{j}$ is the following:

$$
\mathbf{i}_{j}^{R}=\sum_{k \in \Omega_{j}^{+}} \mathbf{i}_{j k}^{R}-\sum_{l \in \Omega_{j}^{-}} \mathbf{i}_{j l}^{R}
$$

where the symbol $\mathbf{i}_{j}^{R}$ indicates the reference injection vector, while $\Omega_{j}^{+}$and $\Omega_{j}^{-}$are the set of outgoing and incoming branches of node $j$, respectively. Focusing on the generic phase $p$, the constraint (13) can be rewritten in its real and imaginary parts as a function of the measurements and the unknowns. Equations similar to (6) are used (also for the injected current) and the same assumptions as before to obtain:

$$
I_{j, p}^{r}-\sum_{k \in \Omega_{j}^{+}} I_{j k, p}^{r}-\sum_{l \in \Omega_{j}^{-}} I_{j l, p}^{r}
$$




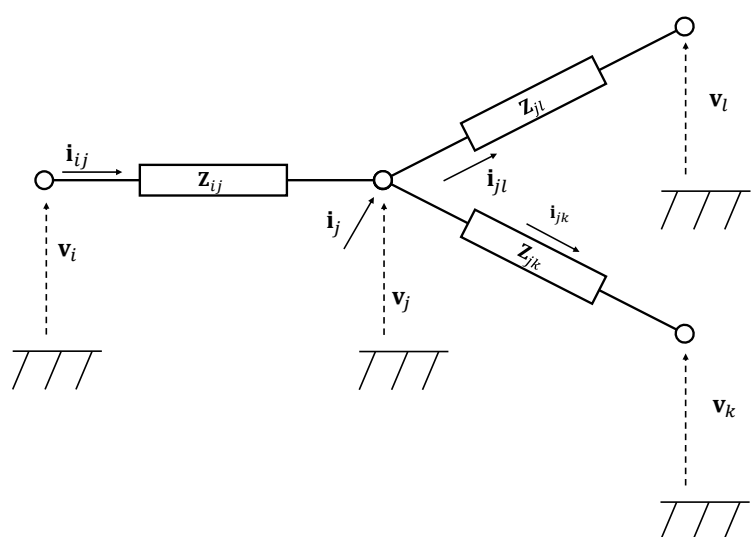

Fig. 2. Multiple branches three-phase example model.

$$
\begin{gathered}
=I_{j, p}^{r}\left(\eta_{j, p}^{s y s}+\eta_{j, p}^{r n d}\right)-I_{j, p}^{x}\left(\psi_{j, p}^{s y s}+\psi_{j, p}^{r n d}\right) \\
-\sum_{k \in \Omega_{j}^{+}} I_{j k, p}^{r}\left(\eta_{j k, p}^{s y s}+\eta_{j k, p}^{r n d}\right)+\sum_{l \in \Omega_{j}^{-}} I_{j l, p}^{r}\left(\eta_{j l, p}^{s y s}+\eta_{j l, p}^{r n d}\right) \\
+\sum_{k \in \Omega_{j}^{+}} i_{j k, p}^{x}\left(\psi_{j k, p}^{s y s}+\psi_{j k, p}^{r n d}\right)-\sum_{l \in \Omega_{j}^{-}} i_{j l, p}^{x}\left(\psi_{j l, p}^{s y s}+\psi_{j l, p}^{r n d}\right)
\end{gathered}
$$

$$
\begin{aligned}
& I_{j, p}^{x}-\sum_{k \in \Omega_{j}^{+}} I_{j k, p}^{x}-\sum_{l \in \Omega_{j}^{-}} I_{j l, p}^{x} \\
& =I_{j, p}^{x}\left(\eta_{j, p}^{s y s}+\eta_{j, p}^{r n d}\right)+I_{j, p}^{r}\left(\psi_{j, p}^{s y s}+\psi_{j, p}^{r n d}\right) \\
& -\sum_{k \in \Omega_{j}^{+}} i_{j k, p}^{x}\left(\eta_{j k, p}^{s y s}+\eta_{j k, p}^{r n d}\right)+\sum_{l \in \Omega_{j}^{-}} i_{j l, p}^{x}\left(\eta_{j l, p}^{s y s}+\eta_{j l, p}^{r n d}\right) \\
& -\sum_{k \in \Omega_{j}^{+}} i_{j k, p}^{r}\left(\psi_{j k, p}^{s y s}+\psi_{j k, p}^{r n d}\right)+\sum_{l \in \Omega_{j}^{-}} i_{j l, p}^{r}\left(\psi_{j l, p}^{s y s}+\psi_{j l, p}^{r n d}\right)
\end{aligned}
$$

where $\eta_{j, p}^{s y s}$ and $\psi_{j, p}^{s y s}$ are the systematic errors of the measured current injection, while the corresponding random errors are denoted by the superscript ${ }^{r n d}$.

The above equations for all the phases can be rewritten in matrix form as:

$$
\begin{aligned}
\mathbf{b}_{j} & =\left[\begin{array}{ll}
\mathbf{H}_{j *}^{\eta, \psi} & \mathbf{H}_{j}^{\eta, \psi}
\end{array}\right]\left[\begin{array}{l}
\boldsymbol{\eta}_{j *}^{\text {sys }} \\
\boldsymbol{\Psi}_{j *}^{\text {sys }} \\
\boldsymbol{\eta}_{j}^{\text {sys }} \\
\boldsymbol{\Psi}_{j}^{\text {sys }}
\end{array}\right]+\left[\begin{array}{ll}
\mathbf{H}_{j *}^{\eta, \psi} & \mathbf{H}_{j}^{\eta, \psi}
\end{array}\right]\left[\begin{array}{c}
\boldsymbol{\eta}_{j *}^{r n d} \\
\boldsymbol{\Psi}_{j *}^{r n d} \\
\boldsymbol{\eta}_{j}^{r n d} \\
\boldsymbol{\Psi}_{j}^{r n d}
\end{array}\right] \\
& =\mathbf{H}_{j} \mathbf{x}_{j}+\boldsymbol{\epsilon}_{j}
\end{aligned}
$$

where all the symbols have a similar meaning as in (10), with $j *$ indicating that all the branches arriving and leaving $j$ must be considered and subscript $j$ referring to the injection quantities. $\mathbf{x}_{j}$ includes all the unknowns associated with both the involved branch and node current measurements.

When a node is a zero-injection node, the information can be used to define constraints similar to the injection constraints. The only difference is that no injection-related unknowns are present and thus the current balance in (14) is a zero vector, while (14) and (15) can be simplified by dropping the injection terms.

\section{Solution of the Estimation Problem}

To solve the estimation problem all the above constraints must be considered and an overall problem can be defined:

$$
\begin{aligned}
\mathbf{b}_{t} & =\mathbf{H}_{t} \mathbf{x}+\boldsymbol{\epsilon}_{t}=\mathbf{H}_{t} \mathbf{x}+\mathbf{H}_{t}^{\xi, \alpha, \eta, \psi} \mathbf{e}_{t} \\
& =\left[\mathbf{H}_{t}^{\xi, \alpha} \mathbf{H}_{t}^{\eta, \psi} \mathbf{H}_{t}^{\gamma, \beta}\right] \mathbf{x}+\left[\mathbf{H}_{t}^{\xi, \alpha} \mathbf{H}_{t}^{\eta, \psi}\right] \mathbf{e}_{t}
\end{aligned}
$$

where the subscript $t$ makes the measurement timestamp explicit, $\mathbf{x}$ is the $N$-size vector of all the unknowns and $\mathbf{e}_{t}$ is the vector of all the zero-mean random errors. $\mathbf{e}_{t}$ has a covariance matrix $\boldsymbol{\Sigma}_{\mathbf{e}}$ (time independent), which is assumed to be diagonal if it includes the variances of decorrelated PMU measurements. If this is not the case, the fundamentals of this method should not be affected, though the covariance matrix would become block diagonal. No limitation prevents the inclusion of correlation information between different measurements, when available. $\boldsymbol{\epsilon}_{t}$ is thus a zero mean random vector and its covariance matrix is obtained as follows:

$$
\boldsymbol{\Sigma}_{\mathbf{\epsilon}_{t}}=\mathbf{H}_{t}^{\xi, \alpha, \eta, \psi} \boldsymbol{\Sigma}_{\mathbf{e}_{t}}\left(\mathbf{H}_{t}^{\xi, \alpha, \eta, \psi}\right)^{\mathrm{T}}
$$

It is interesting to highlight that (17) gives the covariance matrix of the random components in (16) once the constraints are defined as functions of the measured values. Given the measurements, $\mathbf{H}_{t}$ and $\mathbf{H}_{t}^{\xi, \alpha, \eta, \psi}$ are defined and known. The random term represents thus the a posteriori equivalent random deviations of the constraints due to unknown random errors isolated from the other errors.

Additional constraints can be added when considering prior information on the errors and on the line parameter uncertainty (see [17] for details). The initial prior is given by $\mathbf{x}_{p r}=\mathbf{0}_{N \times 1}$ and the associated covariance matrix $\boldsymbol{\Sigma}_{p r}$ is diagonal and given by squared uncertainties associated with the parameters.

Thanks to the high reporting rates of PMUs, which are very fast with respect to typical network dynamics, it is possible to have repeated measurements of the same operating conditions (e.g. considering the meausurements received in a second). The assumption is that over such a small interval the conditions of the specific measured quantity are almost stationary. Even in a DN scenario, small-timescale variations can usually be neglected. However, a check is always performed for the compatibility of the collected measurements, so that abrupt variations can be easily detected. The method allows using a different number of repeated measurements and discarding measurement sets that cannot be relied on, so that the above assumption can be practically met. These measurements can be used to define a problem like (16) for each time instant $t_{i}$. Since these repeated measurements can be considered as repetitions of the same measurement process, the following equivalent problem is considered (using the timestamps $\left.t_{1}, \cdots, t_{M}\right)$ :

$$
\overline{\mathbf{b}}=\overline{\mathbf{H}} \mathbf{x}+\overline{\boldsymbol{\epsilon}}
$$

where $\overline{\mathbf{b}}=\sum_{t=t_{1}}^{t_{M}} \mathbf{b}_{t} / M$ is the average of the equivalent measurement vectors and $\overline{\mathbf{H}}$ and $\overline{\boldsymbol{\epsilon}}$ are the averages of the matrices $\mathbf{H}_{t_{i}}$ and of the vectors $\boldsymbol{\epsilon}_{t}$, respectively. The covariance matrix of $\overline{\boldsymbol{\epsilon}}$, with the above assumptions, can be obtained 
approximately as:

$$
\boldsymbol{\Sigma}_{\overline{\mathbf{e}}}=\frac{1}{M} \overline{\mathbf{H}}^{\xi, \alpha, \eta, \psi} \boldsymbol{\Sigma}_{\mathbf{e}}\left(\overline{\mathbf{H}}^{\xi, \alpha, \eta, \psi}\right)^{\mathrm{T}}
$$

with an analogous interpretation of symbols as before. A proof of the validity of (18) as an approximate, but faster to solve, counterpart of the complete batch WLS, which would involve all the subproblems (16) associated with the timestamps $t_{1}, \cdots, t_{M}$, is given in Appendix $\mathrm{B}$.

The solution of the systematic deviation estimation problem can be improved by considering not only repeated measurements of the same operating conditions but also measurements performed at different times, when the network loading conditions have changed. We can consider $M$ repeated measurements of $P$ different operating conditions (referred to as "cases" in the following). All the equations and defined problems share the same unknown vector $\mathbf{x}$. This means that the systematic error parameters are considered constant among different conditions. This assumptions is further discussed in Section III-A. In this paper, we propose to estimate $\mathbf{x}$ with a sequential procedure that solves multiple WLS problems of type (18), one for each case considered.

The following steps are performed:

1) Solve the problem for the first case $c_{1}$ :

$$
\begin{aligned}
\overline{\mathbf{g}}_{c_{1}} & =\left[\begin{array}{c}
\overline{\mathbf{b}}_{c_{1}} \\
\mathbf{0}
\end{array}\right]=\left[\begin{array}{c}
\overline{\mathbf{H}}_{c_{1}} \\
\mathbf{I}_{N}
\end{array}\right] \mathbf{x}+\left[\begin{array}{c}
\overline{\boldsymbol{\epsilon}}_{c_{1}} \\
\mathbf{e}_{p r}
\end{array}\right] \\
& =\left[\begin{array}{c}
\overline{\mathbf{H}}_{c_{1}} \\
\mathbf{I}_{N}
\end{array}\right] \mathbf{x}+\left[\begin{array}{cc}
\overline{\mathbf{H}}_{c_{1}}^{\xi, \alpha, \eta, \psi} & \mathbf{0} \\
\mathbf{0} & \mathbf{I}_{N}
\end{array}\right]\left[\begin{array}{c}
\overline{\mathbf{e}}_{c_{1}} \\
\mathbf{e}_{p r}
\end{array}\right]=\overline{\mathbf{A}}_{c_{1}} \mathbf{x}+\mathbf{d}_{c_{1}}
\end{aligned}
$$

where $c_{1}$ indicates the first considered case with its associated $M$ repeated measurement sets. The solution is obtained via WLS as:

$\hat{\mathbf{x}}_{c_{1}}=\left(\overline{\mathbf{A}}_{c_{1}}^{T} \mathbf{W}_{c_{1}} \overline{\mathbf{A}}_{c_{1}}\right)^{-1} \overline{\mathbf{A}}_{c_{1}}^{T} \mathbf{W}_{c_{1}} \overline{\mathbf{g}}_{c_{1}}=\mathbf{G}_{c_{1}}^{-1} \overline{\mathbf{H}}_{c_{1}}^{T} \mathbf{W}_{c_{1}} \overline{\mathbf{g}}_{c_{1}}$

using the weighting matrix $\mathbf{W}_{c_{1}}=\left[\begin{array}{cc}\boldsymbol{\Sigma}_{\overline{\mathbf{\epsilon}}_{c_{1}}}^{-1} & \mathbf{0} \\ \mathbf{0} & \boldsymbol{\Sigma}_{p r}^{-1}\end{array}\right]$.

The covariance matrix associated with the estimation is $\boldsymbol{\Sigma}_{\hat{\mathbf{x}}_{c_{1}}}=\mathbf{G}_{c_{1}}^{-1}$.

2) Define the new problem for the new case $c_{2}$ relying on the obtained estimations of case $c_{1}$ :

$$
\overline{\mathbf{g}}_{c_{2}}=\left[\begin{array}{c}
\overline{\mathbf{b}}_{c_{2}} \\
\hat{\mathbf{x}}_{c_{1}}
\end{array}\right]=\left[\begin{array}{c}
\overline{\mathbf{H}}_{c_{2}} \\
\mathbf{I}_{M}
\end{array}\right] \mathbf{x}+\left[\begin{array}{c}
\overline{\mathbf{\epsilon}}_{c_{2}} \\
\mathbf{e}_{\hat{\mathbf{x}}_{c_{1}}}
\end{array}\right]=\overline{\mathbf{A}}_{c_{2}} \mathbf{x}+\mathbf{d}_{c_{2}}
$$

where $\mathbf{e}_{\hat{\mathbf{x}}_{c_{1}}}$ is the estimation error after first step. The estimation is again obtained by WLS considering the weigthing matrix $\mathbf{W}_{c_{2}}=\left[\begin{array}{cc}\boldsymbol{\Sigma}_{\overline{\mathbf{e}}_{c_{2}}}^{-1} & \mathbf{0} \\ \mathbf{0} & \mathbf{G}_{c_{1}}\end{array}\right]$.

The estimation of $\hat{\mathbf{x}}_{c_{2}}$ and of $\boldsymbol{\Sigma}_{\hat{\mathbf{x}}_{c_{2}}}$ are obtained. The problem in (22) can be rewritten considering the deviation from the previously estimated quantities, thus strengthening the linearization of Sections II-B and II-C.

3) Repeat the second step for any new case $c_{i+1}$ using the output of case $c_{i}$ along with its covariance matrix as input data untile all $P$ cases are treated.

The sequential method allows to obtain estimation for each new set of measurements and to update previous estimations accordingly. The overall algorithm is thus a mixed recursive

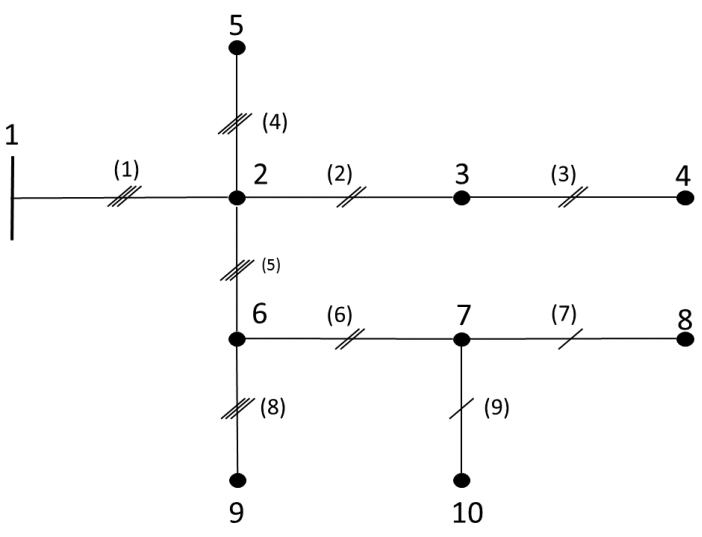

Fig. 3. Three-phase test network.

WLS considering $P$ approximate problems of type (18). When a new estimation is computed, it is possible to check for the presence of bad data (highly erroneous measurements). The WLS paradigm can be exploited by means of a $\chi$-square test detection [20] with a possible normalized residual computation for bad data identification. Other strategies could also be adopted, but new proposals or in-depth analyses on this topic are beyond the scope of the paper.

\section{TESTS AND RESULTS}

\section{A. Test Assumptions}

The proposed methodology has been tested by means of simulations using a sample DN derived from the IEEE 13 bus test network [21]. The network schema is shown in Fig. 3 with node and branch (between parentheses) indices. The network used for the tests has ten nodes and nine branches: four threephase, three two-phase and two single-phase. The network has a zero injection at node 7 . Appendix $\mathrm{C}$ illustrates the branch and load configurations.

Monte Carlo simulations have been performed to verify the performance of the estimator. $N_{M C}=1000$ trials have been used, considering, for each trial, $P=10$ cases and $M=10$ repeated measurements for each case. Each trial corresponds to a different realization of possible systematic errors and of line parameter deviations. A powerflow with reference values is used to compute reference quantities to be measured. Shunt impedances have been duly used in the powerflow even if, as discussed above, they are not present in the estimation model. Preliminary tests have shown that their contribution is practically negligible.

In the following, for the sake of a simpler presentation and without losing generality, we assume that systematic contributions are due mainly to ITs and thus depend on their accuracy class, while random errors are mainly associated with PMUs and thus are included in the accuracy intervals defined by instrument specifications. The approach is in principle able to deal with random contributions arising also from ITs, provided that suitable information is available.

In each trial, for each case and for every set of measurements, systematic and random errors are applied as a cascade (without the first order approximation of (4)) to both 
magnitude and phase angle of the reference synchrophasors obtained with powerflow. As discussed in Section II, the unknown systematic errors in each trials are assumed constant. While for VTs the assumption practically matches the actual behavior, since the voltage variations are limited, for CTs the approximation is looser. In this concern, the proposed estimation algorithm aims to compensate the main systematic contributions associated with the transducers, using a linear model. Residual nonlinearities are thus not included and not compensated. However, the algorithm is practically implemented including a verification of the current levels so that very low currents with respect to nominal ones, where the effects of possible nonlinearities would be more evident, are not considered in the estimation process. The same assumptions as in [18] are adopted to have a realistic setup:

1) Voltage Transformers (VTs) and Current Transformers (CTs) are assumed to be of class 0.5 [22], [23], thus using: $0.5 \%$ for voltage and current ratio errors, $0.6 \mathrm{crad}$ for VT phase-angle displacement and $0.9 \mathrm{crad}$ for CT phase-angle displacement. The errors and deviations, here and in the following, have uniform distributions, meaning a standard deviation $\sigma=\Delta / \sqrt{3}$, where $\Delta$ represents the corresponding maximum absolute deviation. These distributions are used to define each MC trial and thus the actual systematic errors for each estimation process.

2) As for PMUs, a maximum Total Vector Error (TVE) of $0.1 \%$ has been considered by splitting it into a maximum amplitude error of $0.1 \%$ and a maximum phase angle error of $0.1 \mathrm{crad}\left(10^{-3} \mathrm{rad}\right)$.

3) Maximum deviations of line parameters $R_{i j, p q}$ and $X_{i j, p q}$ (for every $(i, j) \in \Gamma$ and $p, q \in\{a, b, c\}$ ) have been assumed equal to $\pm 15 \%$.

4) To simulate different cases, which correspond to different loading conditions, the loads can vary up to $30 \%$. Similar load variations are used in the literature, e.g. in [13] and [24], and can be considered realistic when compared to typical daily load profiles. It is interesting to notice that current variations can be smaller in the main feeders. In the simulations, the voltage profile remains always within the permitted range.

\section{B. Performance Indexes}

To assess the performance, the following root mean square errors (RMSEs) are used:

$$
\begin{aligned}
\operatorname{RMSE}_{\xi_{i, p}^{s y s}} & =\sqrt{\sum_{i=1}^{N_{M C}} \frac{\left(\hat{\xi}_{i, p}^{s y s}-\xi_{i, p}^{s y s}\right)^{2}}{N_{M C}}} \\
\operatorname{RMSE}_{\alpha_{i, p}^{s y s}} & =\sqrt{\sum_{i=1}^{N_{M C}} \frac{\left(\hat{\alpha}_{i, p}^{s y s}-\alpha_{i, p}^{s y s}\right)^{2}}{N_{M C}}} \\
\operatorname{RMSE}_{\eta_{i j, p}^{s y s}} & =\sqrt{\sum_{i=1}^{N_{M C}} \frac{\left(\hat{\eta}_{i j, p}^{s y s}-\eta_{i j, p}^{s y s}\right)^{2}}{N_{M C}}}
\end{aligned}
$$

$$
\begin{aligned}
\operatorname{RMSE}_{\psi_{i j, p}^{s y s}} & =\sqrt{\sum_{i=1}^{N_{M C}} \frac{\left(\hat{\psi}_{i j, p}^{s y s}-\psi_{i j, p}^{s y s}\right)^{2}}{N_{M C}}} \\
\operatorname{RMSE}_{\eta_{k, p}^{s y s}} & =\sqrt{\sum_{i=1}^{N_{M C}} \frac{\left(\hat{\eta}_{k, p}^{s y s}-\eta_{j, p}^{s y s}\right)^{2}}{N_{M C}}} \\
\operatorname{RMSE}_{\psi_{j, p}^{s y s}} & =\sqrt{\sum_{i=1}^{N_{M C}} \frac{\left(\hat{\psi}_{k, p}^{s y s}-\psi_{j, p}^{s y s}\right)^{2}}{N_{M C}}}
\end{aligned}
$$

where denotes the estimated quantities, $p$ the analysed phase, $(i, j) \in \Gamma$, and $k$ indicates the measured injection node as in Section II. RMSEs thus represent a statistical index of the errors between the actual and estimated systematic errors.

\section{Single branch}

If only a single branch is considered (branch $(1,2)$, nodes 1 and 2), the results in terms of $\xi_{i, p}^{s y s}$ and $\alpha_{i, p}^{s y s}$ are reported in Table I for the three-phases. The RMSE results are compared with the standard deviations (between parentheses) of the extracted systematic errors computed in the $N_{M C}$ trials (which are close to the theoretical values derived from the assumed accuracies dividing by $\sqrt{3}$ ). These standard deviations thus represent the prior RMSEs. The improvements can be seen from percentages of RMSE reduction reported under each value. The results concerning $\operatorname{RMSE}_{\eta_{12, p}^{s y s}}$ and $\operatorname{RMSE}_{\psi_{12, p}^{s y s}}$ are not shown in Table I, since the improvements are very small (respectively below $0.4 \%$ and $1 \%$ ).

TABLE I

RMSE of Estimated Voltage Magnitude and Phase-Angle SYSTEMATIC ERRORS FOR BRANCH 1 (SINGLE-BRANCH ESTIMATION).

\begin{tabular}{c|cccc}
\hline \multirow{2}{*}{ Phase } & \multicolumn{4}{|c}{ RMSE [\%] } \\
& $\xi_{1, p}^{\text {sys }}$ & $\xi_{2, p}^{\text {sys }}$ & $\alpha_{1, p}^{\text {sys }}$ & $\alpha_{2, p}^{\text {sys }}$ \\
\hline \multirow{3}{*}{$\mathrm{a}$} & 0.21 & 0.21 & 0.25 & 0.25 \\
& $(0.28)$ & $(0.29)$ & $(0.35)$ & $(0.36)$ \\
& $-26 \%$ & $-29 \%$ & $-27 \%$ & $-29 \%$ \\
\hline \multirow{2}{*}{$\mathrm{b}$} & 0.21 & 0.21 & 0.25 & 0.25 \\
& $(0.29)$ & $(0.29)$ & $(0.35)$ & $(0.35)$ \\
& $-28 \%$ & $-27 \%$ & $-29 \%$ & $-27 \%$ \\
\hline \multirow{2}{*}{$\mathrm{c}$} & 0.21 & 0.22 & 0.25 & 0.25 \\
& $(0.29)$ & $(0.29)$ & $(0.34)$ & $(0.34)$ \\
& $-26 \%$ & $-27 \%$ & $-27 \%$ & $-25 \%$ \\
\hline
\end{tabular}

To assess the validity of the first order approximation and of the procedure presented in Section II-D, the results in Table I have been compared with those obtained with a full batch WLS implementation using Gauss-Newton iterative solution of the nonlinear estimation problem defined without approximations. The percent RMSE values are almost the same (only the fourth decimal digit changes), while the execution times with the proposed approach are about one order of magnitude lower. Similar results are obtained for all the tests in the following, achieving an empirical proof of the correctness of the assumptions made in Section II to define the proposed technique for the estimation problem. 


\section{Multiple branches}

Another set of tests has been performed to verify the performance obtained when the algorithm is applied to a set of branches simultaneously. Figures 4-9 refer to the systematic error estimation obtained using the full network when only voltage drop constraints are considered (compared with standard deviations of extracted errors as before). Figures 4 and 5 show, respectively, the estimation RMSE of the voltage related parameters for phase $a$ as a function of the node index (node 3, 4 and 8 are missing since they are two-phase nodes without the first phase). It is clear that a strong reduction of the systematic errors (about $60 \%$, that is much larger than in single-branch case) can be achieved for both magnitude and phase-angle by compensating the measurements with the estimated parameters. Similar results can be found for phases $b$ and $c$. If $30 \%$ maximum deviation is assumed for the line parameters (tests not shown here for the sake of brevity), the results worsen only slightly, thus showing the efficacy of the method also when such parameters are known with high uncertainty.

The RMSE values give a statistical index of the estimation performance achieved. To give a better insight, Fig. 6 compares, for all the MC trials and with reference to the first case of each trial, the values of node 2 voltage magnitude affected by VT systematic error and the corresponding values after that error has been compensated using the estimated $\hat{\xi}_{2, a}^{\text {sys }}$. The compensated voltages are clearly distributed in a narrower band around the reference value. All the reference values have been normalized to one to allow an easier comparison and thus the horizontal dashed line represents the perfect compensation. Complementary information is also given by the representations of the errors in Fig. 7. In the upper graph the histograms of actual systematic errors and residual errors after voltage magnitude compensation are compared for the same node 2 . The occurrence frequency for each $0.05 \%$-wide bin is reported. In the lower graph, the same errors are plotted in terms of their empirical cumulative distributions (cdf). The residual errors distribution depends on the distributions of the systematic errors, but it is also the result of the combinations due to the estimation algorithm. It is clear how the errors are more frequently located around lower values and within a smaller range.

As in the single-branch case, different results are obtained when focusing on branch currents. Figure 8 shows the results in terms of $\mathrm{RMSE}_{\eta_{i j, a}^{s y s}}$ for all the branches of phase $a$, while

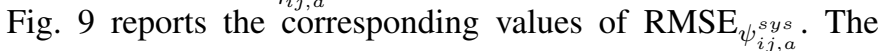
advantages, even if present, are very limited (an error reduction of less than $1 \%$ for $\eta_{i j, a}^{s y s}$ and at most $2 \%$ for $\psi_{i j, a}^{s y s}$. With a $30 \%$ maximum deviation of the line parameters the reductions are even smaller (e.g. almost halved for $\operatorname{RMSE}_{\psi_{i j, a}^{s y s}}$ ).

On the contrary, when current injection measurements are also present, $\operatorname{RMSE}_{\eta_{i j, a}^{s y s}}$ and $\mathrm{RMSE}_{\psi_{i j, a}^{s y s}}$ undergo strong reductions (up to $57 \%$ and $62 \%$, respectively) at some branches, as shown in Figs. 10 and 11. The constraints directly applied to the currents thus limit the variability of the systematic errors in the measurements of the involved or near branches. This is also confirmed by tests performed with higher variability of

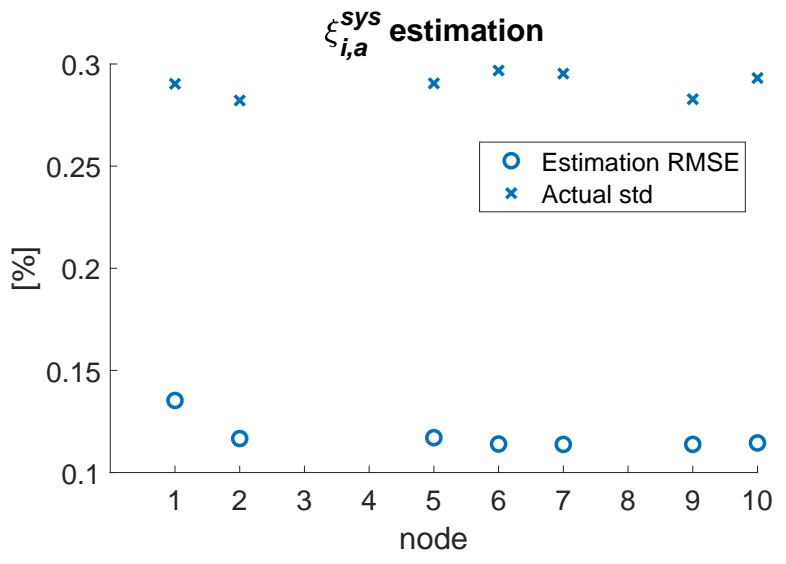

Fig. 4. RMSE of estimated voltage magnitude systematic errors, see eq. (23).

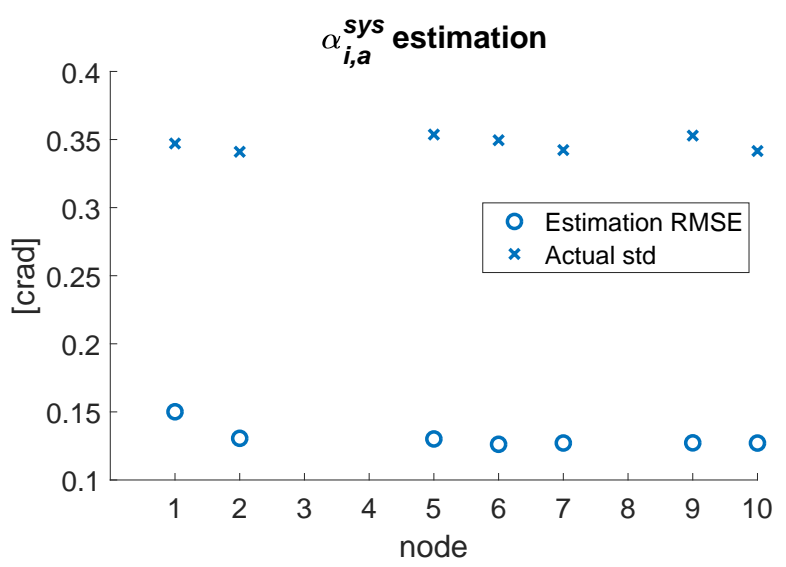

Fig. 5. RMSE of estimated voltage phase-angle systematic errors, see eq. (24)

the line parameters $(30 \%)$, since the results are very similar. The effects of the additional constraints on $\mathrm{RMSE}_{\xi_{i, p}^{s y s}}$ and $\mathrm{RMSE}_{\alpha_{i, p}^{s y s}}$ are instead negligible in this configuration.

When injections are measured (nodes 2, 3, 6 are considered in the following), the state is extended to include the corresponding magnitude and phase-angle systematic errors $\eta_{j, p}^{s y s}$ and $\psi_{j, p}^{s y s}$. Table II compares the $\operatorname{RMSE}_{\eta_{j, p}^{s y s}}$ and $\operatorname{RMSE}_{\psi_{j, p}^{s y s}}$ for nodes 3 and 6 with the initial uncertainty of the parameters (be-

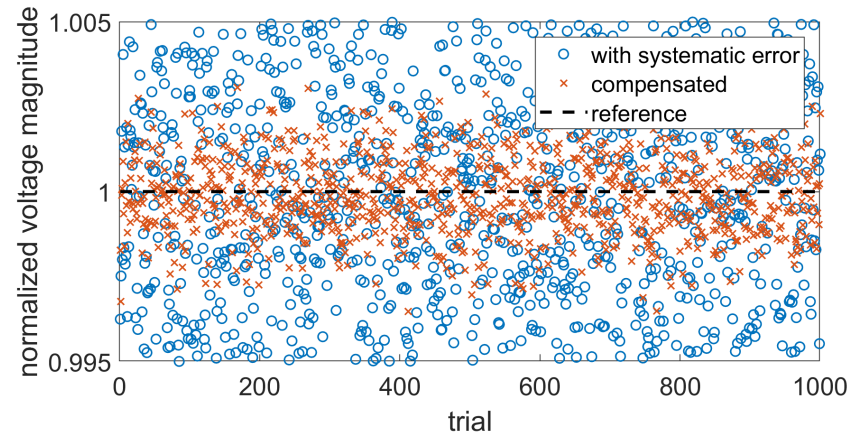

Fig. 6. Normalized voltage magnitudes in the presence of systematic errors before and after compensation. 

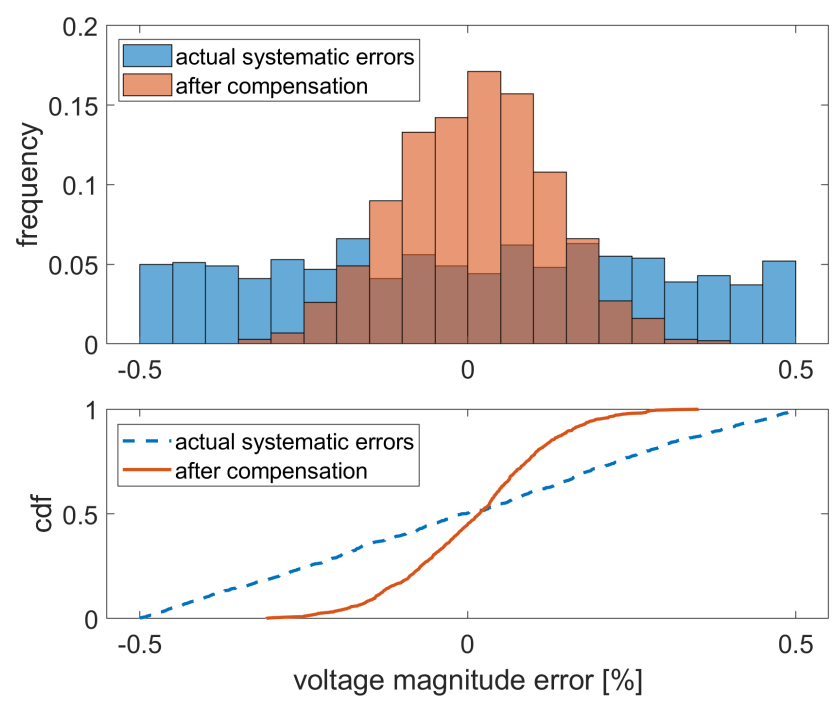

Fig. 7. Systematic error compensation: histograms of systematic errors before and after compensation (top graph); empirical cumulative distribution of the errors (bottom graph).

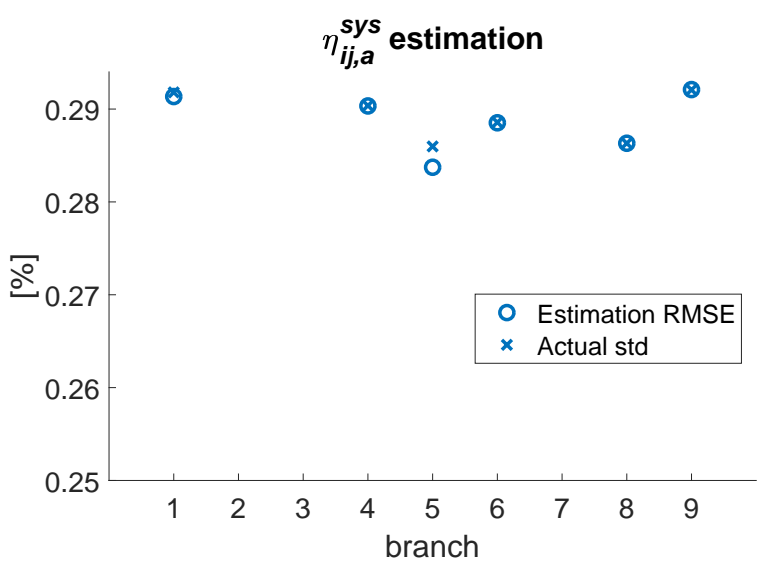

Fig. 8. RMSE of estimated line current magnitude systematic errors, see eq. (25).

tween parentheses) for all the three-phases. The improvements are clear (percentages of reduction are reported in the third row of each block). Node 2 does not achieve significant advantages and the RMSEs are almost equal to prior uncertainties.

TABLE II

RMSE of Estimated INJECTED CURRENT MAGNitude AND PhaSE-ANGLE SyStEMATIC ERRORS.

\begin{tabular}{c|cccccc}
\hline $\begin{array}{c}\text { Injection } \\
\text { Node }\end{array}$ & RMSE [\%] \\
& $\eta_{j, a}^{\text {sys }}$ & $\eta_{j, b}^{\text {sys }}$ & $\eta_{j, c}^{\text {sys }}$ & $\psi_{j, a}^{\text {sys }}$ & $\psi_{j, b}^{\text {sys }}$ & $\psi_{j, c}^{\text {sys }}$ \\
\hline \multirow{3}{*}{3} & - & 0.16 & 0.16 & - & 0.25 & 0.27 \\
& $(-)$ & $(0.29)$ & $(0.29)$ & $(-)$ & $(0.52)$ & $(0.52)$ \\
& - & $-44 \%$ & $-46 \%$ & - & $-53 \%$ & -48 \\
\hline \multirow{2}{*}{6} & 0.15 & 0.13 & 0.13 & 0.22 & 0.24 & 0.21 \\
& $(0.29)$ & $(0.29)$ & $(0.29)$ & $(0.52)$ & $(0.52)$ & $(0.52)$ \\
& $-47 \%$ & $-54 \%$ & $-53 \%$ & $-57 \%$ & $-53 \%$ & $-60 \%$ \\
\hline
\end{tabular}

Other tests have been performed considering zero-injection at node 7 as well. In Fig. 12, the $\mathrm{RMSE}_{\eta_{i j, p}^{s y s}}$ values are reported

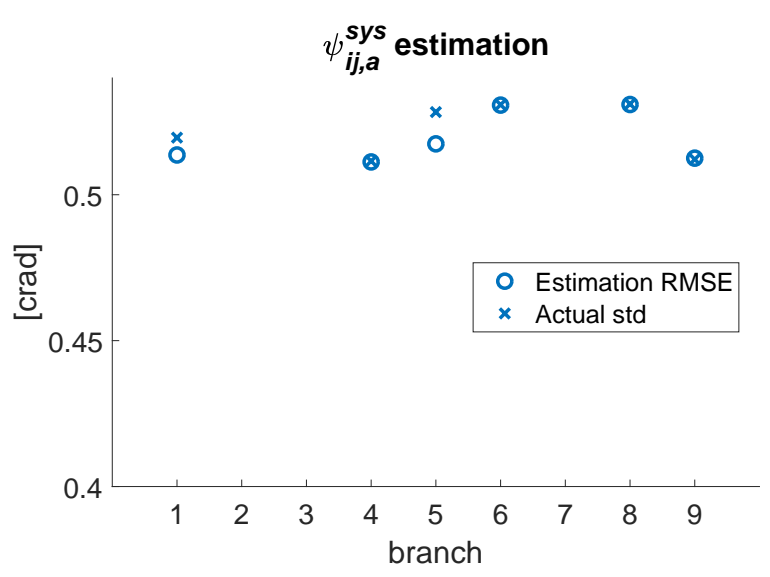

Fig. 9. RMSE of estimated line current phase-angle systematic errors, see eq. (26).

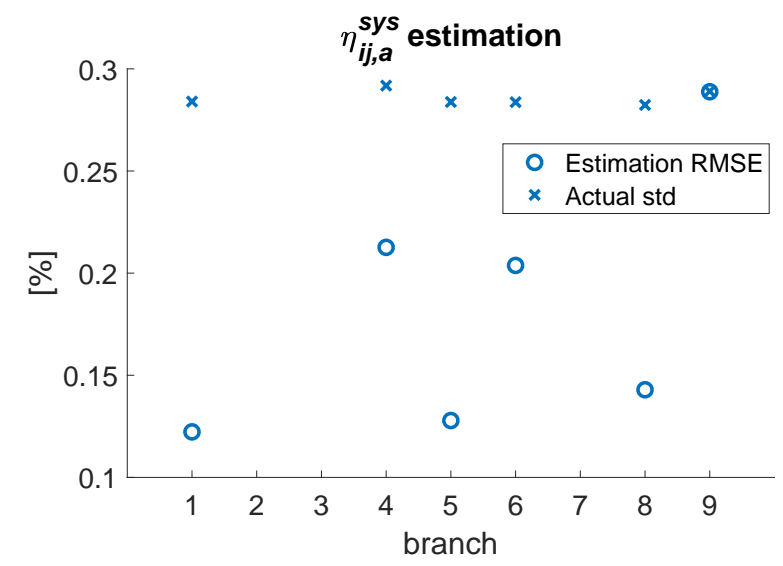

Fig. 10. RMSE of estimated line current magnitude systematic errors when constraints also on injected currents are considered, see eq. (27).

(and compared with the standard deviations of the corresponding parameters), showing that the zero-injection constraints significantly improve the estimation for the involved branches of phase $a$ and $c$ (RMSE reductions up to $30 \%$ and $26 \%$, respectively), while phase $c$ is almost unaffected since it is not present in branches 6,7 and 9. Similar results have been obtained for $\mathrm{RMSE}_{\psi_{i j, p}^{s y s}}$ with a maximum reduction of about $31 \%$.

As a final consideration, it has been verified that the WLS problem conditioning does not degrade significantly when multiple branches and injection constraints are introduced (constraints are not virtual measurements as in other applications). This, among others, is the effect of a homogeneous accuracy of the instruments across the network and of the lack of extremely different lengths and impedances among the branches. If very large DNs with highly problematic topologies were considered, the method could be still applied to set of branches while multi-area strategies could be introduced.

\section{Conclusions}

A method for estimating and compensating the systematic errors of synchronized phasor measurements in three-phase 


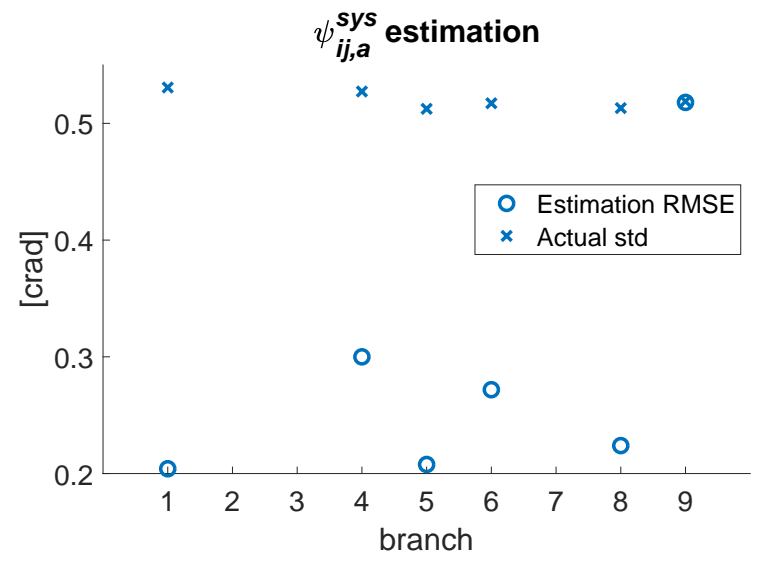

Fig. 11. RMSE of estimated line current phase-angle systematic errors when constraints also on injected currents are considered, see eq. (28).

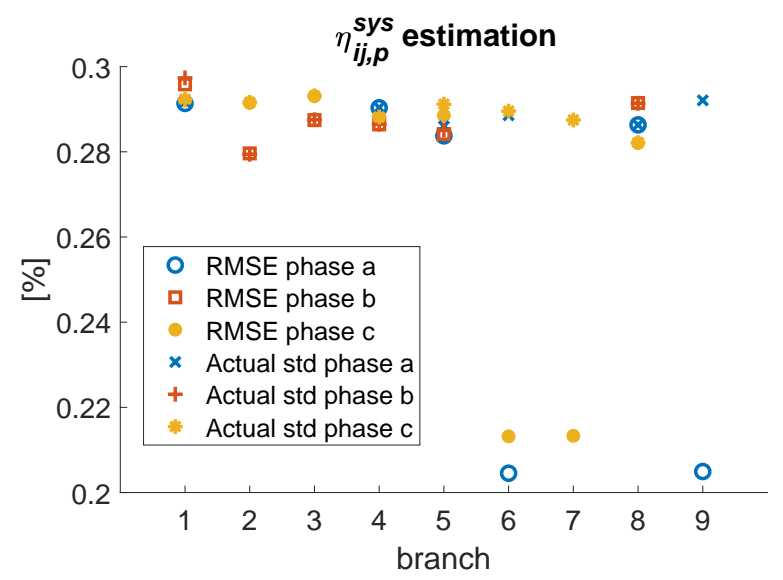

Fig. 12. RMSE of the estimations of line current magnitude deviations with zero-injection constraints.

distribution networks has been presented. The estimation algorithm takes into account the different uncertainty contributions in the measurement chain and considers line parameter uncertainty in the measurement model as well.

Testing has shown that additional constraints, such as additional injection measurements and zero-injections, allow a much better estimation of current measurement systematic errors.

The proposed techique is a first step towards the definition of a total estimator that allows for a self-aware monitoring system that is able to continuously improve its data.

\section{ApPENDiX A}

Measurement Matrices of the Three-Phase Line Voltage Drop Estimation Problem

The expressions of the measurement matrices in the problem (10) are given in the full page equations (A.1), (A.2) and (A.3).

\section{APPENDIX B}

\section{Proof of the Validity of Repeated Measurement EQUIVALENT PROBLEM}

The solution of problems like (20) and (22) (the latter in the same form as the former) is obtained from a type (21) solution. Considering a generic case associated with matrix $\overline{\mathbf{A}}=\left[\begin{array}{c}\overline{\mathbf{H}} \\ \mathbf{I}_{N}\end{array}\right]$ and equivalent measurements $\overline{\mathbf{g}}=\left[\begin{array}{l}\overline{\mathbf{b}} \\ \mathbf{0}\end{array}\right]$ (the subscript referring to a specific case is, from here on, dropped for the sake of simplicity), where overbars denote that they are averages of $M$ timestamp-specific counterparts $\mathbf{A}_{t_{i}}$ and $\mathbf{g}_{t_{i}}$. Since these matrices are linear functions of the measurements (see (A.1)(A.3)) averaging corresponds to compute the matrices from the average measurements. The random error vector is $\overline{\boldsymbol{\epsilon}}=$ $\overline{\mathbf{H}}^{\xi, \alpha, \eta, \psi} \overline{\mathbf{e}}$. The solution of the problems is:

$$
\hat{\mathbf{x}}=\left(\overline{\mathbf{H}}^{T} \boldsymbol{\Sigma}_{\overline{\mathbf{e}}}^{-1} \overline{\mathbf{H}}+\boldsymbol{\Sigma}_{\mathbf{e}_{p r}}^{-1}\right)^{-1} \overline{\mathbf{H}}^{T} \boldsymbol{\Sigma}_{\overline{\mathbf{e}}} \overline{\mathbf{b}}
$$

In the following, it is proven that the estimate in (B.1) is equivalent, from a practical viewpoint, to the solution of the following problem:

$\left[\begin{array}{c}\mathbf{b}_{t_{1}} \\ \vdots \\ \mathbf{b}_{t_{M}} \\ \mathbf{0}\end{array}\right]=\left[\begin{array}{c}\mathbf{H}_{t_{1}} \\ \vdots \\ \mathbf{H}_{t_{M}} \\ \mathbf{I}_{N}\end{array}\right] \mathbf{x}+\left[\begin{array}{cccc}\mathbf{H}_{t_{1}}^{\xi, \alpha, \eta, \psi} & \mathbf{0} & \cdots & \mathbf{0} \\ \vdots & \ddots & & \vdots \\ & & \mathbf{H}_{t_{N}}^{\xi, \alpha, \eta, \psi} & \mathbf{0} \\ \mathbf{0} & \cdots & \mathbf{0} & \mathbf{I}_{N}\end{array}\right]\left[\begin{array}{c}\boldsymbol{\epsilon}_{t_{1}} \\ \vdots \\ \boldsymbol{\epsilon}_{t_{M}} \\ \mathbf{e}_{p r}\end{array}\right]$

which is:

$$
\hat{\mathbf{x}}^{\prime}=\left(\sum_{i=1}^{M} \mathbf{H}_{t_{i}}^{T} \boldsymbol{\Sigma}_{\boldsymbol{\epsilon}_{t_{i}}}^{-1} \mathbf{H}_{t_{i}}+\boldsymbol{\Sigma}_{\mathbf{e}_{p r}}^{-1}\right)^{-1} \sum_{i=1}^{M} \mathbf{H}_{t_{i}}^{T} \boldsymbol{\Sigma}_{\boldsymbol{\epsilon}_{t_{i}}}^{-1} \mathbf{b}_{t_{i}}
$$

Focusing on $\overline{\mathbf{W}}=\boldsymbol{\Sigma}_{\overline{\mathbf{\epsilon}}}^{-1}$ and defining $\delta \mathbf{F}_{t_{i}} \triangleq \mathbf{H}_{t_{i}}^{\xi, \alpha, \eta, \psi}-$ $\mathbf{H}_{R}^{\xi, \alpha, \eta, \psi}$ (subscript $R$ indicates here and in the following matrices and vectors computed from the reference values), we can write:

$$
\begin{aligned}
\overline{\mathbf{W}}= & {\left[\frac{\sum_{i=1}^{M}\left(\mathbf{H}_{R}^{\xi, \alpha, \eta, \psi}+\delta \mathbf{F}_{t_{i}}\right)}{M} \frac{\boldsymbol{\Sigma}_{\mathbf{e}}}{M}\right.} \\
& \left.\times \frac{\sum_{i=1}^{M}\left(\mathbf{H}_{R}^{\xi, \alpha, \eta, \psi^{\mathrm{T}}}+\delta \mathbf{F}_{t_{i}}^{\mathrm{T}}\right)}{M}\right]^{-1} \\
\simeq & {\left[\mathbf{H}_{R}^{\xi, \alpha, \eta, \psi} \boldsymbol{\Sigma}_{\mathbf{e}} \mathbf{H}_{R}^{\xi, \alpha, \eta, \psi^{\mathrm{T}}}+\left(\sum_{i=1}^{M} \delta \mathbf{F}_{t_{i}}\right) \boldsymbol{\Sigma}_{\mathbf{e}} \mathbf{H}_{R}^{\xi, \alpha, \eta, \psi}{ }^{\mathrm{T}}\right.} \\
& \left.+\mathbf{H}_{R}^{\xi, \alpha, \eta, \psi} \boldsymbol{\Sigma}_{\mathbf{e}} \sum_{i=1}^{M} \delta \mathbf{F}_{t_{i}}^{\mathrm{T}}\right]^{-1}=M\left(\boldsymbol{\Sigma}_{R}+\delta \tilde{\boldsymbol{\Sigma}}\right)^{-1} \\
\simeq & M\left(\boldsymbol{\Sigma}_{R}^{-1}-\boldsymbol{\Sigma}_{R}^{-1} \delta \tilde{\boldsymbol{\Sigma}} \boldsymbol{\Sigma}_{R}^{-1}\right)=M\left(\mathbf{W}_{R}+\delta \tilde{\mathbf{W}}\right)
\end{aligned}
$$

where the approximations are obtained by neglecting second order terms. Similarly, defining $\delta \mathbf{H}_{t_{i}} \triangleq \mathbf{H}_{t_{i}}-\mathbf{H}_{R}$, it is possible to obtain:

$$
\begin{aligned}
\mathbf{W}_{t_{i}} & \simeq\left(\boldsymbol{\Sigma}_{R} \delta \mathbf{F}_{t_{i}} \boldsymbol{\Sigma}_{\mathbf{e}} \mathbf{H}_{R}^{\xi, \alpha, \eta, \psi^{\mathrm{T}}}+\mathbf{H}_{R}^{\xi, \alpha, \eta, \psi} \boldsymbol{\Sigma}_{\mathbf{e}} \delta \mathbf{F}_{t_{i}}^{\mathrm{T}}\right)^{-1} \\
& =\left(\boldsymbol{\Sigma}_{R}+\delta \boldsymbol{\Sigma}_{t_{i}}\right)^{-1} \simeq \boldsymbol{\Sigma}_{R}-\boldsymbol{\Sigma}_{R}^{-1} \delta \boldsymbol{\Sigma}_{t_{i}} \boldsymbol{\Sigma}_{R}^{-1} \\
& =\mathbf{W}_{R}+\delta \mathbf{W}_{t_{i}}
\end{aligned}
$$




$$
\begin{gathered}
\mathbf{H}_{i j}^{\xi, \alpha}=\left[\begin{array}{cccc}
V_{i, a}^{r} & -V_{i, a}^{r} & -V_{i, a}^{x} & +V_{j, a}^{x} \\
V_{i, a}^{x} & -V_{i, a}^{x} & V_{i, a}^{r} & V_{j, a}^{r} \\
\vdots & \vdots & \vdots & \vdots
\end{array}\right], \\
\mathbf{H}_{i j}^{\eta, \psi}=\left[\begin{array}{cccc}
-R_{i j, a a}^{0} I_{i j, a}^{r}+X_{i j, a a}^{0} I_{i j, a}^{x} & +R_{i j, a a}^{0} I_{i j, a}^{x}+X_{i j, a a}^{0} I_{i j, a}^{r} & \cdots \\
-R_{i j, a a}^{0} I_{i j, a}^{x}-X_{i j, a a}^{0} I_{i j, a}^{r} & -R_{i j, a a}^{0} I_{i j, a}^{r}+X_{i j, a a}^{0} I_{i j, a}^{x} & \cdots \\
\vdots & \vdots
\end{array}\right], \\
\mathbf{H}_{i j}^{\gamma, \beta}=\left[\begin{array}{ccc}
R_{i j, a a}^{0} I_{i j, a}^{r} & -X_{i j, a a}^{0} I_{i j, a}^{x} & \cdots \\
R_{i j, a a}^{0} I_{i j, a}^{x} & +X_{i j, a a}^{0} I_{i j, a}^{r} & \cdots \\
\vdots & \vdots &
\end{array}\right],
\end{gathered}
$$

Substituting (B.4) and $\overline{\mathbf{b}}=\sum_{i=1}^{M} \mathbf{b}_{t_{i}} / M$ in (B.1) and defining $\delta \mathbf{b}_{t_{i}} \triangleq \mathbf{b}_{t_{i}}-\mathbf{b}_{R}$, after a few passages, we obtain:

$$
\begin{aligned}
\hat{\mathbf{x}} \simeq & {\left[M \mathbf{H}_{R}^{T} \mathbf{W}_{R} \mathbf{H}_{R}+\left(\sum_{i=1}^{M} \delta \mathbf{H}_{t_{i}}^{\mathrm{T}}\right) \mathbf{W}_{R} \mathbf{H}_{R}\right.} \\
& \left.+\mathbf{H}_{R} \mathbf{W}_{R} \sum_{i=1}^{M} \delta \mathbf{H}_{t_{i}}+M \mathbf{H}_{R}^{T} \delta \tilde{\mathbf{W}} \mathbf{H}_{R}+\mathbf{\Sigma}_{\mathbf{e}_{p r}}^{-1}\right]^{-1} \\
& \times\left[M \mathbf{H}_{R}^{T} \mathbf{W}_{R} \mathbf{b}_{R}+\left(\sum_{i=1}^{M} \delta \mathbf{H}_{t_{i}}\right) \mathbf{W}_{R} \mathbf{b}_{R}\right. \\
& \left.+\mathbf{H}_{R}^{T} \mathbf{W}_{R} \sum_{i=1}^{M} \delta \mathbf{b}_{t_{i}}+M \mathbf{H}_{R}^{T} \delta \tilde{\mathbf{W}} \mathbf{b}_{R}\right]
\end{aligned}
$$

and we can compare the estimation with the following (from (B.3), using (B.5)):

$$
\begin{aligned}
\hat{\mathbf{x}}^{\prime} \simeq & {\left[M \mathbf{H}_{R}^{T} \mathbf{W}_{R} \mathbf{H}_{R}+\left(\sum_{i=1}^{M} \delta \mathbf{H}_{t_{i}}^{\mathrm{T}}\right) \mathbf{W}_{R} \mathbf{H}_{R}\right.} \\
& \left.+\mathbf{H}_{R} \mathbf{W}_{R} \sum_{i=1}^{M} \delta \mathbf{H}_{t_{i}}+\mathbf{H}_{R}^{T}\left(\sum_{i=1}^{M} \delta \mathbf{W}_{t_{i}}\right) \mathbf{H}_{R}+\mathbf{\Sigma}_{\mathbf{e}_{p r}}^{-1}\right]^{-1} \\
& \times\left[M \mathbf{H}_{R}^{T} \mathbf{W}_{R} \mathbf{b}_{R}+\sum_{i=1}^{M} \delta \mathbf{H}_{t_{i}} \mathbf{W}_{R} \mathbf{b}_{R}\right. \\
& \left.+\mathbf{H}_{R}^{T} \mathbf{W}_{R} \sum_{i=1}^{M} \delta \mathbf{b}_{t_{i}}+\mathbf{H}_{R}^{T}\left(\sum_{i=1}^{M} \delta \mathbf{W}_{t_{i}}\right) \mathbf{b}_{R}\right]
\end{aligned}
$$

Looking at (B.6) and (B.7), the only differences are in the terms depending on the variations of the weighting matrices (the fourth term for each pair of square brackets in both equations). But, considering (B.4) and (B.5), it is possible to see that $\delta \tilde{\mathbf{W}}=\sum_{i=1}^{M} \delta \mathbf{W}_{t_{i}}$ and thus, $\mathbf{x} \simeq \mathbf{x}^{\prime}$ as claimed.

\section{APPENDIX C}

\section{Test Network Line Configuration And DATA}

Figure 3 reports the test network topology along with the branch indexes. Branches 1 and 5 correspond to configuration 601 (with length 2000 feets) of the line data in [25] (document IEEE 13 Node Test Feeder.doc), while branches 2, 3 correspond to configuration 603 (lengths 500 and 300 feets, respectively) and branches $4,6,7,8,9$ to configurations 602 ,
604, 605, 606 and 607 respectively (lengths 500, 300, 300, 500, 800 feets, respectively).

TABLE III

NOMINAL POWERS OF THE LOADS.

\begin{tabular}{c|c|cccccccc}
\hline $\begin{array}{c}\text { Power } \\
\text { type }\end{array}$ & Ph & \multicolumn{10}{|c}{ Node } \\
\hline \multirow{3}{*}{ P [kW] } & $\mathrm{a}$ & 8.5 & 0 & 0 & 160 & 393.5 & 0 & 485 & 128 \\
& $\mathrm{~b}$ & 33 & 170 & 230 & 120 & 418 & 0 & 68 & 0 \\
& $\mathrm{c}$ & 58.5 & 170 & 230 & 120 & 613.5 & 170 & 290 & 0 \\
\hline \multirow{3}{*}[\mathbf{kVAr}]{} & $\mathrm{a}$ & 5 & 0 & 0 & 110 & 225 & 0 & -10 & 86 \\
& $\mathrm{~b}$ & 19 & 125 & 132 & 90 & 239 & 0 & -140 & 0 \\
& $\mathrm{c}$ & 34 & 125 & 132 & 90 & 405 & -120 & 12 & 0 \\
\hline
\end{tabular}

\section{ACKNOWLEDGEMENTS}

The work of P. Castello, C. Muscas and P. A. Pegoraro described in this paper has been partially funded within the R\&D project Cagliari2020 (partially funded by the Italian University and Research Ministry, grant MIUR-PON04a200381).

\section{REFERENCES}

[1] AA. VV., Phasor Measurement Units and Wide Area Monitoring Systems, 1st ed., A. Monti, C. Muscas, and F. Ponci, Eds. Academic Press, 2016.

[2] S. Chakrabarti, E. Kyriakides, and M. Albu, "Uncertainty in power system state variables obtained through synchronized measurements," IEEE Transactions on Instrumentation and Measurement, vol. 58, no. 8 , pp. 2452-2458, Aug. 2009.

[3] F. Aminifar, M. Shahidehpour, M. Fotuhi-Firuzabad, and S. Kamalinia, "Power system dynamic state estimation with synchronized phasor measurements," IEEE Transactions on Instrumentation and Measurement, vol. 63, no. 2, pp. 352-363, Feb. 2014.

[4] M. Asprou, E. Kyriakides, and M. M. Albu, "Uncertainty bounds of transmission line parameters estimated from synchronized measurements," IEEE Transactions on Instrumentation and Measurement, pp. 1-11, 2018, online pre-print. [Online]. Available: https://ieeexplore.ieee.org/document/8472294.

[5] V. Milojević, S. Čalija, G. Rietveld, M. V. Ačanski, and D. Colangelo, "Utilization of PMU measurements for three-phase line parameter estimation in power systems," IEEE Transactions on Instrumentation and Measurement, vol. 67, no. 10, pp. 2453-2462, Oct 2018.

[6] D. Ritzmann, P. S. Wright, W. Holderbaum, and B. Potter, "A method for accurate transmission line impedance parameter estimation," IEEE Transactions on Instrumentation and Measurement, vol. 65, no. 10, pp. 2204-2213, 2016.

[7] A. von Meier, E. Stewart, A. McEachern, M. Andersen, and L. Mehrmanesh, "Precision micro-synchrophasors for distribution systems: A summary of applications," IEEE Transactions on Smart Grid, vol. 8, no. 6, pp. 2926-2936, Nov. 2017. 
[8] M. Pau, P. A. Pegoraro, and S. Sulis, "Efficient branch-current-based distribution system state estimation including synchronized measurements," IEEE Transactions on Instrumentation and Measurement, vol. 62, no. 9, pp. 2419-2429, Sep. 2013.

[9] S. Sarri, L. Zanni, M. Popovic, J. L. Boudec, and M. Paolone, "Performance assessment of linear state estimators using synchrophasor measurements," IEEE Transactions on Instrumentation and Measurement, vol. 65, no. 3, pp. 535-548, March 2016.

[10] A. Mingotti et al., "Calibration of synchronized measurement system: from the instrument transformer to the pmu," in 2018 IEEE 9th International Workshop on Applied Measurements for Power Systems (AMPS), Sept 2018, pp. 1-5.

[11] P. Tosato et al., "A tuned lightweight estimation algorithm for low-cost phasor measurement units," IEEE Transactions on Instrumentation and Measurement, vol. 67, no. 5, pp. 1047-1057, May 2018.

[12] M. Pignati et al., "Real-time state estimation of the EPFL-campus medium-voltage grid by using PMUs," in 2015 IEEE Power Energy Society Innovative Smart Grid Technologies Conference (ISGT), Feb. 2015, pp. 1-5.

[13] Z. Wu, L. T. Zora, and A. G. Phadke, "Simultaneous transmission line parameter and PMU measurement calibration," in PES General Meeting, IEEE, 2015, pp. 1-5.

[14] A. Pal, P. Chatterjee, J. S. Thorp, and V. A. Centeno, "Online calibration of voltage transformers using synchrophasor measurements," IEEE Transactions on Power Delivery, vol. 31, no. 1, pp. 370-380, Feb 2016.

[15] X. Wang et al., "Online calibration of phasor measurement unit using density-based spatial clustering," IEEE Transactions on Power Delivery, vol. 33, no. 3, pp. 1081-1090, June 2018.

[16] C. Roberts et al., "Improving distribution network model accuracy using impedance estimation from micro-synchrophasor data," in PES General Meeting (PESGM), 2016, pp. 1-5.

[17] P. A. Pegoraro, K. Brady, P. Castello, C. Muscas, and A. von Meier, "Line impedance estimation based on synchrophasor measurements for power distribution systems," IEEE Transactions on Instrumentation and Measurement, pp. 1-12, 2018, online pre-print. [Online]. Available: https://ieeexplore.ieee.org/document/8444098.

[18] R. Puddu, K. Brady, C. Muscas, P. A. Pegoraro, and A. V. Meier, "PMUbased technique for the estimation of line parameters in three-phase electric distribution grids," in 2018 IEEE 9th International Workshop on Applied Measurements for Power Systems (AMPS), Sept 2018, pp. $1-5$.

[19] C. Muscas, M. Pau, P. Pegoraro, and S. Sulis, "Effects of measurements and pseudomeasurements correlation in distribution system state estimation," IEEE Trans. Instrum. Meas., vol. 63, no. 12, pp. 2813-2823, Dec. 2014.

[20] A. Abur and A. G. Expòsito, Power System State Estimation. Theory and Implementation. Marcel Dekker, New York, 2004.

[21] R. Abu-Hashim et al., "Test systems for harmonics modeling and simulation," IEEE Trans. Power Del., vol. 14, no. 2, pp. 579-587, Apr 1999.

[22] Instrument transformers - Part 2: Inductive voltage transformers, IEC Int. Std. IEC 60044-2, 1997.

[23] Instrument transformers - Part 1: Current transformers, IEC Int. Std. IEC 60044-1, 2003.

[24] A. Gómez-Expósito, C. Gómez-Quiles, and I. Džafić, "State estimation in two time scales for smart distribution systems," IEEE Transactions on Smart Grid, vol. 6, no. 1, pp. 421-430, Jan 2015.

[25] IEEE PES AMPS DSAS Test Feeder Working Group. 13bus feeder data. [Online]. Available: http://sites.ieee.org/pestestfeeders/files/2017/08/feeder13.zip 